\title{
A New Stochastic Radiation Antenna Array Based on Frequency Hopping with Different Chip-Rates
}

\author{
Yuanyue Guo $^{1+}$, Dongjin Wang ${ }^{2}$ and Yong-Sheng Zhou ${ }^{3}$ \\ ${ }^{1}$ Key Laboratory of Electromagnetic Space Information, Chinese Academy of Sciences \\ ${ }^{2}$ University of Science and Technology of China \\ ${ }^{3}$ Academy of Opto-Electronics, Chinese Academy of Sciences
}

\begin{abstract}
In order to enhance its temporal-spatial stochastic characteristics of stochastic radiation fields, a new microwave staring correlated imaging method based on random frequency hopping with different chiprates (MSCI-RFH-DCR) is proposed, which is characterized that microwave pulse signals emitted respectively by different elements of stochastic radiation radar array (SRRA) have the same pulse periods and repetition frequencies, however, each pulse contains multiple chips of random frequency hopping with different chip-rates and orthogonal frequency hopping patterns each other. The imaging model is established, then the process of stochastic radiation fields excitated by the SRRA with random frequency hopping with different chip-rates in the target observation area is investigated in detail, meanwhile its temporal-spatial stochastic characteristics is analyzed; Finally, numerical simulations is demonstrated that compared with random frequency hopping with the same chip-rates, the present method can achieve better temporal-spatial stochastic characteristics of stochastic radiation fields in the condition of limited signal bandwidth and lower chip-rates of frequency hopping, and by the low-speed sampling of scattered echo signals, a high-resolution microwave imaging can be obtained, which can reduce the cost and complexity of imaging system.
\end{abstract}

Keywords: microwave staring correlated imaging, random frequency hopping with different chip-rates, temporal-spatial stochastic characteristics of radiation fields, super-resolution

\section{Introduction}

Traditional microwave staring imaging is a kind of radar imaging method to obtain target information of its electromagnetic scattering characteristics based on stationary or quasi-stationary platform [1], which has its advantages to perform remote sensing monitoring of important targets in a fixed region in all-day and allweather, without limitation of light illumination and cloud occlusion, but its imaging resolution is limited by its antenna aperture of radar [2].

Recently microwave staring correlated imaging has been proposed [3], by random multiple antenna radiation with multiple phase centres and correlated reconstruction algorithm, which can break the limitation of antenna aperture to obtain a high resolution imaging. However, the scheme has to face a serious challenge to its application, which a high resolution imaging requires a great quantity of samplings of stochastic radiated fields, and its huge data processing of correlated imaging. For microwave staring correlated imaging, the ideal temporal-spatial stochastic characteristics of the radiated fields is a prerequisite for its realization, which indicates its non-correlation properties between radiated fields at different resolution cells, as well as between different time in the whole observation area. Main factors which affect its temporal-spatial stochastic characteristics include pulse signal forms emitted by SRRA, its frequency bandwidth, chip-rates and its spatial arrangement of antenna array etc.

\footnotetext{
Corresponding author. Tel.: +86-13866135598; fax: +(86-551-63603942).

E-mail address: yuanyueg@ustc.edu.cn.
} 
In its project realization of microwave correlated imaging, under the conditions of limited antenna array scale, limited bandwidth, lower chip-rates (less than $100 \mathrm{Mc} / \mathrm{s}$ ) of random pulse signal forms, it is still a key technical problem to design and optimize the SRRA to enhance its temporal-spatial stochastic characteristics of radiated fields.

\section{System Model}

The geometry of microwave staring correlated imaging system is illustrated in Fig. 1. SRRA with multiple elements is fixed in a stationary platform with its aperture D, and the receiver is located at its central position, where $z_{0}$ is the vertical distance between the imaging system platform and imaging area $S(r)$ under antenna beam coverage, meanwhile the center of imaging area $S(r)$ has its downwards angle of observation relative to the platform.

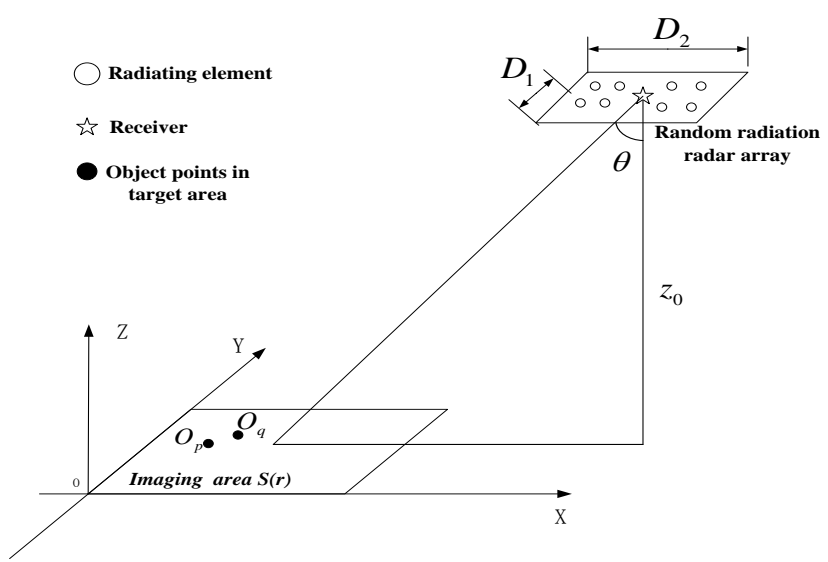

Fig. 1: Microwave staring correlated imaging scene.

Each antenna element from 1 to $N$ transmit respectively microwave pulse signal of random frequency hopping at different chip-rates, whose frequency hopping signal of the $i$-th radiation element at $t$ time can be expressed as:

$$
f\left(r_{i}^{r^{T}}, t\right)=\sum_{m=1}^{M} \sum_{k=1}^{K} r e c t\left(\frac{t-m T_{t}-\frac{2 k+1}{2} T_{d i}}{T_{d i}}\right) \exp \left\{j 2 \pi f_{i, m k}\left(t-m T_{t}-k T_{d i}\right)+j \theta_{i, m k}\right\}
$$

where $i=0,1, \ldots, N-1, N$ is the number of antenna elements, $\stackrel{r}{r}_{i}^{T}$ is the location of its antenna phase center, $T_{t}$ indicates each pulse period of $M$ pulses, which consist of $K$ chips of frequency hopping, where $T_{d i}$ is its chip period; For the $k$-th chip in the $m$-th pulse, $f_{i, m k}$ is its hopping frequency and $\theta_{i, m k}$ is its initial phases.

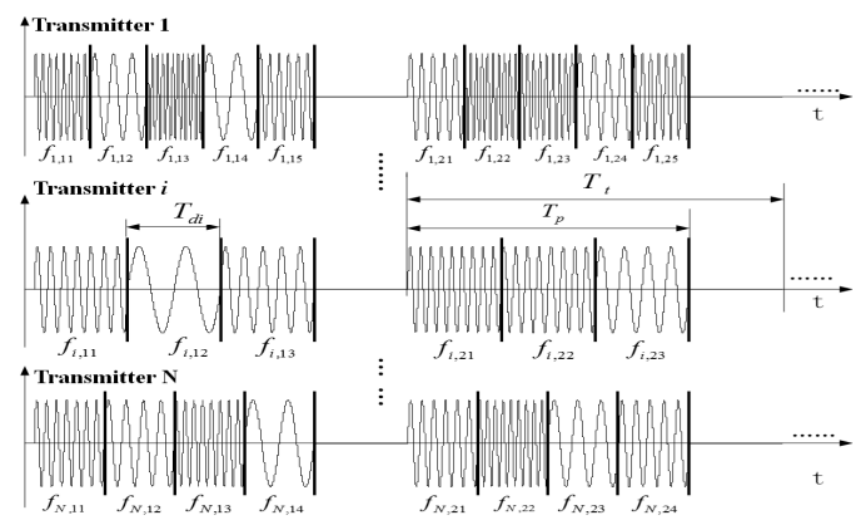

Fig. 2: Random frequency hopping signals with different chip-rates at different antenna elements.

As shown in Fig. 2, for any radiation antenna elements $T_{d i}$ are different from each other, as well as its different chip-rates of frequency hopping. As a selection mode, the difference between chip-rates of any two 
radiation elements can be chosen randomly in a certain range, as a optimization, its values can be prime number each other; In another hand, its frequency hopping patterns are orthogonal for each other.

According to electromagnetic field propagation in free space, stochastic radiated fields in imaging area $S(r)$ produced by above antenna array can be expressed as:

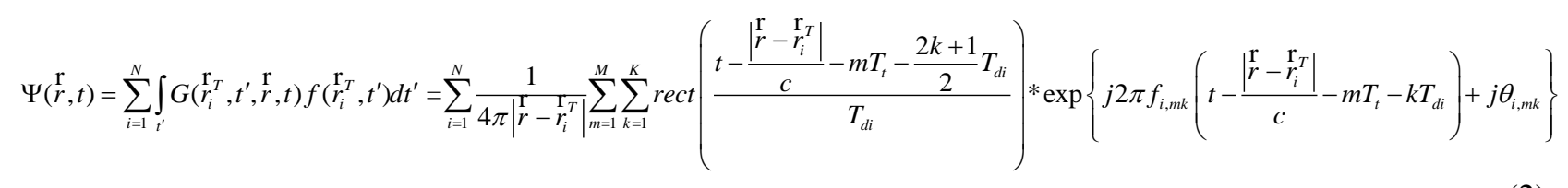

where $c$ is the speed of light and $f\left(r_{i}^{T}, t^{\prime}\right)$ denote the chip signal of random frequency hopping of $i$-th antenna element at its transmission time $t^{\prime}, G\left(r_{i}^{T}, t^{\prime}, r, t\right)$ is the time-domain Green function of the free space, which obeys the following equation [4]:

$$
G\left(r_{i}^{T}, t^{\prime}, \stackrel{r}{r}, t\right)=\frac{\delta\left(t-t^{\prime}-\frac{\left|\stackrel{1}{r}-\stackrel{1}{r}_{i}^{\prime}\right|}{c}\right)}{4 \pi \mid r-r_{r}^{r} r_{i}^{T}}
$$

where $\delta$ is the well-known Dirac-delta function.

At the time $t$, radiated fields at ${ }_{r}{ }_{1}$ and ${ }_{2} r_{2}$ in imaging area $S(r)$ appear its spatial non-correlation characteristics, which can be defined as :

$$
I\left(r_{1}, r_{2}, t\right)=<\Psi^{*}\left(r_{1}, t\right) \Psi\left(r_{2}, t\right)>
$$

Herein, the ensemble average $\langle\cdot\rangle$ indicates inner-product operation, $(\cdot)^{*}$ is the symbol of matrix conjugate transpose.

Meanwhile at different time $t_{1}$ and $t_{2}$, radiated fields on imaging area $S(r)$ have its temporal noncorrelation characteristics, which can be given as :

$$
I\left(t_{1}, t_{2}\right)=\int_{S} \Psi^{*}\left(\stackrel{r}{r}, t_{1}\right) \Psi\left(\stackrel{r}{r}, t_{2}\right) d r
$$

Under the first-order Born approximation, the scattered echo obtained by the receiver at $\vec{r}^{R}$ can be donated as $[5,6]$ :

$$
\operatorname{rec}\left(\stackrel{\mathrm{r}}{r}^{R}, t\right)=\int_{S} \frac{\Psi\left(\mathrm{r} r, t-\frac{\left|\stackrel{\mathrm{I}}{r}^{R}-\stackrel{\mathrm{r}}{r}\right|}{c}\right)}{4 \pi\left|\stackrel{\mathrm{r}}{r}^{R}-\stackrel{\mathrm{r}}{r}\right|} \cdot \sigma(\stackrel{\mathrm{r}}{r}) d S
$$

where $\sigma(r)$ represents the backscattering coefficients distribution of the target scattering centers in the imaging area $S(r)$. It can be seen obviously that all target information are coupled in the received scattering echo signals.

Corresponding to inverse scattering problem in electromagnetic field, Microwave staring correlated imaging can extract the target information $\hat{\sigma}(\boldsymbol{r})$ based on the received echo $\operatorname{rec}\left(r^{R}, t\right)$ and random radiated fields $\Psi(r, t)$, which correlated process can be written as:

$$
\hat{\boldsymbol{\sigma}}(\boldsymbol{r})=\wp\left[\Psi(\stackrel{\mathrm{r}}{r}, t), \operatorname{rec}\left(\stackrel{\mathrm{r}}{r}^{R}, t\right)\right]
$$

where $\wp$ indicates the first-order correlated operator.

\section{Analysis on Stochastic Characteristics of Radiated Fields}

In Fig. 1, stochastic radiated fields are investigated at two locations $\mathrm{O}_{p}=\left(x_{p}, y_{p}, 0\right)$ and $\mathrm{O}_{q}=\left(x_{q}, y_{q}, 0\right)$, shown as two black circles very close to each other in the target area, and it can be considered that the difference of its propagation time delay of each radiating element to the two position is very small: 


$$
\max \left\{\frac{\left|d\left(r_{i}^{T}, \mathrm{O}_{p}\right)-d\left(r_{i}^{T}, \mathrm{O}_{q}\right)\right|}{c}\right\}<<T_{d} \quad(i=1,2, \ldots, N)
$$

Here $d(\cdot, \cdot)$ indicates the Euclidean distance between two points.

While antenna elements emit random frequency hopping signal at the same chip-rates, as well as with the same chip-periods ${ }^{T_{d}}$, and Without loss of generality, the sampling period of the scattered echo signals are given as ${ }^{T_{s}}=T_{d}$.

As shown in Fig. 3, at a sampling time, radiated fields at two scattering points $\mathrm{O}_{p}$ and $\mathrm{O}_{q}$ is the superposition of different samplings of random frequency hopping signals of different array elements.

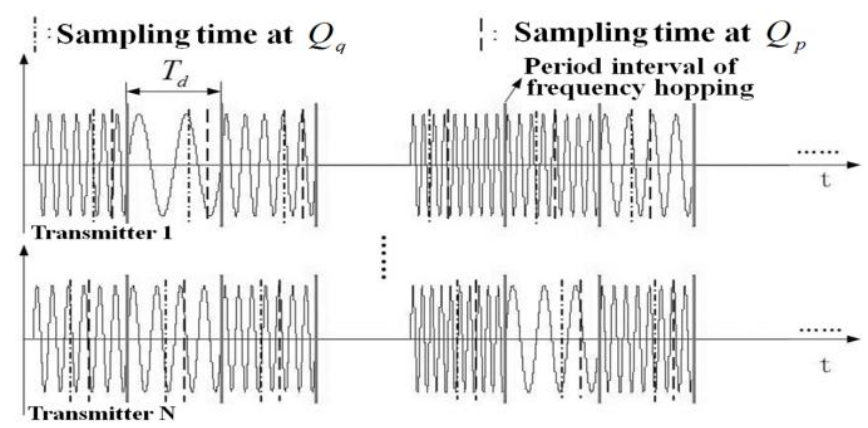

Fig. 3: Sampling of random frequency hopping signal with the same chip-rates at two closer scattering points.

Obviously, because the distance between $\mathrm{O}_{p}$ and $\mathrm{O}_{q}$ is so near that sampling times of each emitting frequency hopping signal at $\mathrm{O}_{p}$ and $\mathrm{O}_{q}$ almost fall in a single chip-period, which will lead to the sampling values of radiated fields distribution at two points are almost uniform, so the two points cannot be resolved.

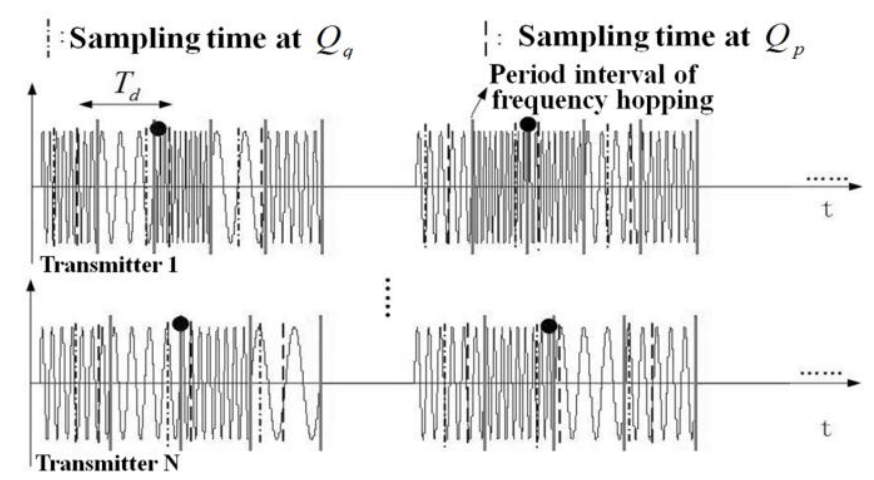

Fig. 4: Sampling of random frequency hopping signal with the different chip-rates at two closer scattering points.

In the proposed scheme, each antenna element emit random frequency hopping signals at different chiprates with different chip-periods $T_{d i}$. As shown in Fig. 4, the sampling time of each emitting frequency hopping signal at $\mathrm{O}_{p}$ and $\mathrm{O}_{q}$ are likely to fall into different chip-periods, which will cause directly different radiation field distributions at the two points, therefore, a possibility to resolve the two points is provided. Consequently, the radiated fields produced by SRRA have better temporal-spatial stochastic characteristics, achieving super-resolution imaging.

\section{Numerical Simulation and Analysis}

By using numerical simulations, the performances of the proposed scheme are demonstrated, including its ability of super-resolution imaging. The scenario for simulation is shown in Fig. 1 with the system simulation parameters bellow. The configuration of antenna array with a rectangular distribution of $5 \times 5$ elements with equal spacing of $0.3 \mathrm{~m}$ has been arranged with its platform height $z_{0}=120 \mathrm{~m}$ and its downwards angle of observation $\theta=45^{\circ}$; Next, the size of image area is $24 m \times 24 m$, and a continuous target model is discretized into $80 \times 80$ grids with Grid size $0.3 m \times 0.3 m$ as depicted in Fig. 5 . 


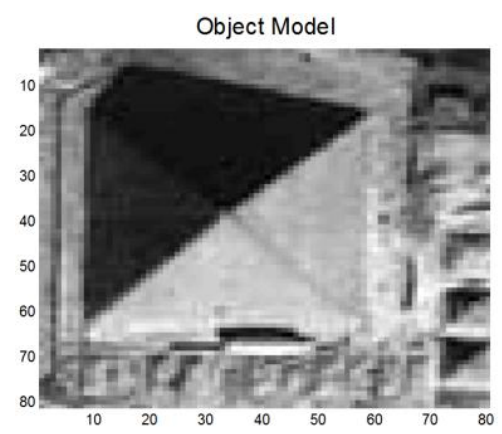

Fig. 5: Target model in simulation.

Three types of signals are investigated in simulation. Signal 1 form as a reference to illustrate the superiority of the present scheme, which have the same chip-rates of random frequency hopping emitted by array elements, and its carrier frequency is $f_{0}=10 \mathrm{GHz}$ with its hopping frequency ranges of $1 \mathrm{GHz}$, and its pulse period is $T_{t}=2.4 \mu \mathrm{s}$ with its chip-period of $T_{d}=40 \mathrm{~ns}$, as well as its duty ratio of $10 \%$. The random hopping frequency patterns are ensured by its orthogonality.

Signal 2 is a kind of random frequency hopping pulse signals with different chip-rates for each radiating element, with its chip-periods $T_{d i}$ vary among $(1-15 \%) T_{d} \sim(1+15 \%) T_{d}$, and other parameter is the same as Signal 1 .

When Signal 2 is used by radiation elements, its radiation field distributions in imaging area $S(r)$ at two different time T1, T2 are shown respectively in Fig. 6 (a) and Fig. 6 (b).
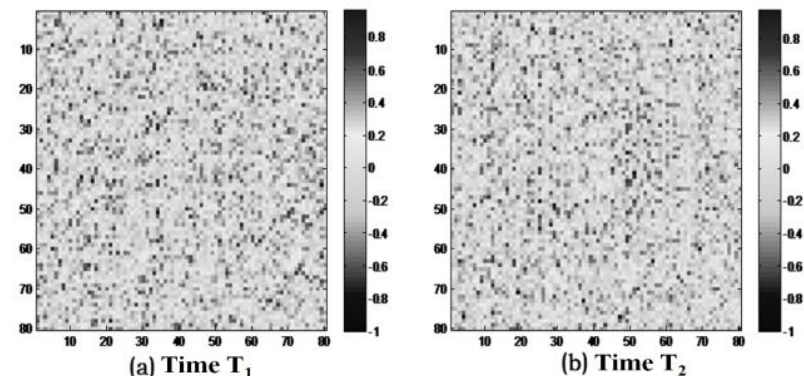

Fig. 6: Stochastic radiation field distribution based on random frequency hopping with different chip-rates.

It is obvious that microwave radiated fields appear better spatial stochastic characteristics in the imaging plane at the same time, as well as temporal stochastic characteristics at different time, in other words consistent with its two-dimensional temporal-spatial stochastic characteristics, which show the effectiveness of Signal 2.

When Signal 1 and Signal 2 are adopted respectively, both sampling periods of scattered echoes are $T_{s}=T_{d}=40 n s$, and both sampling times are 6400, then its temporal and spatial correlation characteristics of corresponding stochastic radiated fields are obtained in Fig. 7(a) and Fig. 8(a), and by correlation imaging process, its imaging results are shown in Fig. 7(b) and Fig. 8(b).
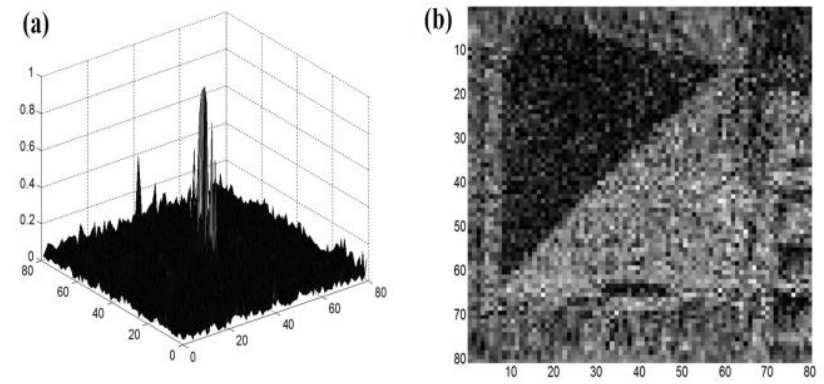

Fig. 7: Spatial correlation characteristics of stochastic radiated fields and imaging result with the same chip-rates.

Comparing Fig. 7 and Fig. 8, it can be found obviously that its peak of the correlation of stochastic radiated fields in the case of Signal $\mathbf{2}$ is sharper, as well as more flat around the peak, which describes its 
greater differences of stochastic radiation field distributions and smaller cross-correlations for any two points in the imaging area region, as well as its better potentiality of imaging resolution at two points. Therefore its imaging result in Fig. 8(b) is better than in Fig. 7(b).
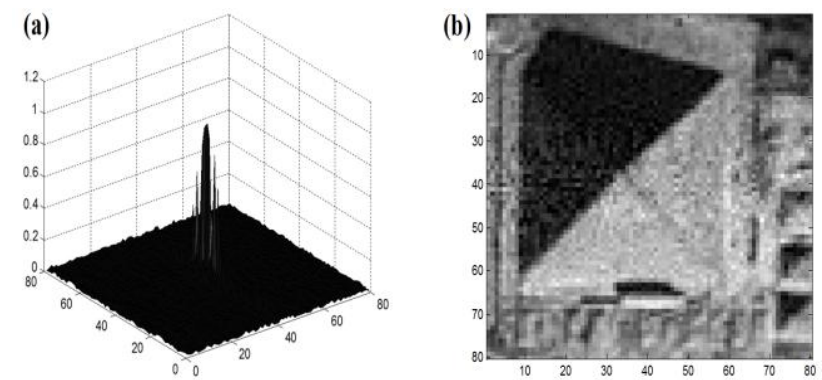

Fig. 8: Spatial correlation characteristics of stochastic radiated fields and imaging result with different chip-rates.

\section{Conclusion}

Based on a type of random frequency hopping signal with different chip-rates, a new stochastic radiation antenna array has been studied in this paper. Imaging model of MSCI-RFH-DCR is founded. By the analysis of its forming process of stochastic radiated fields under the condition of different chip-rates of random frequency hopping, its mechanism to enhance its temporal-spatial stochastic characteristics of radiated fields is described. Compared with the traditional hopping frequency with the same chip-rates, numerical simulation shows that the proposed scheme has its potential to improve its temporal-spatial stochastic characteristics of radiated fields, and the same better imaging results at low-speed emission and low-speed sampling can be achieved, which will reduce the cost and complexity of the imaging system.

\section{Acknowledgements}

This work was supported by the Hi-Tech Research and Development Program of China (Grant Project No.2013AA122903).

\section{References}

[1] Bao Z, Xing M and Wang T. 2005 Radar Imaging Technology (Beijing: Publishing House of Electronics Industry)

[2] Roger J. Sullivan. Radar Foundations for Imaging and Advanced Concepts, SciTech Publishing, Inc., 2004.

[3] Y. Guo, D. Wang, X. He. A novel super-resolution imaging method based on stochastic radiation radar array, Measurement Science and Technology, 2013, 24(7):074013.

[4] Felsen L B and Marcuvitz N. 1994 Radiation and Scattering of Waves (New York: Wiley-IEEE).

[5] Giovanni L, Raffaele P and Rocco P. 1999 Inverse scattering under the distorted Born approximation for cylindrical geometries, Opt. Soc. Am. A 16 1779-87.

[6] Slaney M, Kak A C and Larsen L E. 2009 Limitation of imaging with first-order diffraction tomography IEEE Trans. Microw. Theory Tech. 32 1467-81. 